\title{
Simple Assessment of Lactate Threshold by Means of the Bench Stepping in Older Population
}

\author{
Makoto Ayabe*, Takuya Yahiro ", Yukari Mori***, Kohsaku Takayama*, Takuro Tobina*, \\ Hiroyuki Higuchi $^{* * *}$, Kojiro Ishii", Ichiro Sakuma ${ }^{* * * * *}$, Yutaka Yoshitake ${ }^{* * * * *}$, Hideo Miyazaki ${ }^{* * * * * *}$, \\ Akira Kiyonaga $^{* *}$, Munehiro Shindo**, and Hiroaki Tanaka** \\ *Laboratory of Human Performance and Fitness, Graduate School of Education, Hokkaido University \\ Nisi 7, Kita 11-jo, Kita-ku, Sapporo City, Hokkaido 060-0811 Japan \\ ${ }^{* *}$ Faculty of Sports Sciene, Fukuoka University \\ htanaka@fukuoka-u.ac.jp \\ ${ }^{* * *}$ Graduate School of Physical Education, Fukuoka University \\ 8-19-1, Nanakuma, Jounan-ku, Fukuoka City, Fukuoka 814-0180 Japan \\ ${ }^{* * * *}$ Department of Cardiovascular Medicine, Graduate School of Medicine, Hokkaido University \\ Nishi 7, Kita 15-jo, Kita-ku, Sapppro City, Hokkaido 060-8638 Japan \\ ${ }^{* * * * *}$ Department for Interdisciplinary Studies of Lifelong Sport and Physical Activity, \\ National Institute of Fitness and Sports in Kanoya \\ 1, Shiromizu-cho, Kanoya City, Kagoshima, 891-2393 Japan \\ ${ }^{*}$ Graduate School of Medical and Dental Sciences, Niigata University \\ 5274 Niban-cho, Gakkocho-doori, Niigata City, Niigata 951-8514 Japan \\ [Received January 23, 2003 ; Accepted March 13, 2003]
}

The purpose of this study was to develop a simple assessment of lactate threshold (LT) in older people. Two-hundreds and nine subjects, aged 65 to 90 years old, performed a bench stepping test by means of a $20 \mathrm{~cm}$ bench at 10 to 35 ascends per minute of the stepping rate. In 52 of 209 subjects, we determined the metabolic equivalents corresponding to exercise intensity of which lactate (LA) increased $0.1,0.2,0.3,0.4,0.5$ and $1 \mathrm{mmol} / \mathrm{l}$ from baseline level, LA corresponding to the baseline level and 2 mmol/l (METs@LAr+0.1, METs@LAr+0.2, METs@LAr+0.3, METs@LAr+0.4, METs@LAr+0.5, METs@LAr+1, METs@LAr and METs@LA2), in addition to LT. We calucurated exercise intensity equivalent to fixed LA levels by both a standard aseessment using a liner regression between exercise intensity (logexercise inetsnity) and LA (log-LA) ranged above LT, and a simple assessment using a liner regression between log-exercise inetsnity and log-LA ranged bellow and above $2 \mathrm{mmol} / \mathrm{l}$ of LA. Using the standard assessment, no significant difference was confirmed between METs@LAr+0.1 (4.7 \pm 0.8 METs) and LT (4.6 \pm 0.7 METs). In regard to METs corresponding to other LA, using the standard assessment, METs@LAr (4.5 \pm 0.8 METs) was significantly lower $(p<0.05)$, and the rest $(4.8 \pm 0.8-5.6 \pm 0.8$ METs $)$ were significantly higher $(p<0.01)$, compared with LT. There was no significant difference between METs@LAr+0.1 determined using standard assessment and simple assessment (4.6 \pm 0.9 METs). Also METs@LAr+0.1 determined by the simple assessment had high correlation and good limit agreement with LT $(r=0.900, p<0.001$ and $0.0 \pm 0.8$ METs), as well as METs@LAr+0.1 determined by the standard assessment $(\mathrm{r}=0.930, \mathrm{p}<0.001$ and $0.0 \pm 0.6 \mathrm{METs})$. In $90 \%$ of men and $92 \%$ of women, LA reached $2 \mathrm{mmol} / \mathrm{l}$ at an intensity of 20, 25 or 30 ascends/minute. The method of evaluating exercise intensity corresponding to $0.1 \mathrm{mmol} / \mathrm{l}$ above resting level of LA using a stepping rate of 15, 20, 25 and 30 ascends/minute on a $20 \mathrm{~cm}$ platform, taking $L A$ of $2 \mathrm{mmol} / \mathrm{l}$ as the termination criteria, can be considered valid as a simple assessment of LT in older individuals.

Keywords: elderly, lactate threshold, step test

[International Journal of Sport and Health Science Vol.1 (2), 207-215, 2003]

\section{Introduction}

Appropriate aerobic exercise has been recommended to improve health [ACSM, (2000)]. In Japan, facing 
an aging population, there is a crying social need for positive development of health-improving programs emphasizing exercise. Therefore, we believe that it is an urgent task to develop systems to evaluate aerobic capacity, and to prescribe appropriate exercise based on those evaluations for large numbers of the population.

Compared to other ergometers, the stepping test is a convenient and superior graded exercise test [ACSM, (2000)]. In Canada, the stepping test has become familiar in the household at the individual level as a realistic method of evaluating aerobic capacity [Bell and Jacobs, (1992a, 1992b); Cox et al., (1992)]. Because these methods evaluate aerobic capacity based on heart rate (HR) during and after exercise, although they are convenient, the accuracy may be faulty for the elderly. [Cox, et al., (1992) ; Takeshima et al., (1992)]. Briefly stated, while maximum oxygen uptake $\left(\mathrm{VO}_{2} \max \right)$ estimated by HR response has a $10-15 \%$ of the estimated error in younger individuals, it is deteriorated for older adults, because of the increase in inter-individual variability of maximal HR. Additionally, this method is inapplicable for some older individuals, for reasons such as side effects of medicines that may affect heart response or the use of pacemakers.

Blood lactate concentration (LA) during submaximal exercise is closely related to aerobic capacity, especially, lactate threshold (LT) and OBLA (Onset of Blood Lactate Accumulation) are indicators of aerobic capacity better or at least no worse than $\dot{\mathrm{VO}}_{2} \max$ [Yoshida et al., (1987) ; Tokmakidis et al., (1998)]. Because LT in particular can be measured in light to moderate submaximal exercise, it has possibilities for safe and accurate evaluation, in older people. Furthermore LT can be used directly for the exercise prescription, and the improvement of acerbic capacity, blood pressure and blood lipids have been confirmed as training effects [Kiyonaga et al., (1985) ; Motoyama et al., (1998) ; Sunami et al., (1999)]. However LT has not been generalized, because of necessity for the skilled technique associated with the frequent blood sampling. On the other hand, recently developed portable LA analyser simplified sampling and analysis, and their accuracy has been proven satisfactory [Pyne et al., (2000)]. Thus LA measurement will be able to be used in health promotion fields by means of this devise.

Therefore, present investigation examined the validity of LT determined by LA during a 2-4 stages
Tab. 1. The Characteristics of Subjects

\begin{tabular}{lccc}
\hline & ALL & Men & Women \\
\hline $\mathrm{N}$ & 209 & 106 & 103 \\
Age $($ years $)$ & $72(3)$ & $72(3)$ & $72(4)$ \\
Height $(\mathrm{cm})$ & $156.5(8.5)$ & $162.9(6.1)$ & $149.9(4.9)$ \\
Weight $(\mathrm{kg})$ & $57.2(10.5)$ & $61.9(10.4)$ & $52.3(8.1)$ \\
\hline \multicolumn{3}{c}{ Mean (Standard Deviation) }
\end{tabular}

of step test, using the portable LA analyser, for the purpose of developing a simple assessment of LT, particularly for older people.

\section{Methods}

\subsection{Subjects}

Subjects of this research were 209 subjects, 106 males and 103 females, from 65 to 90 years of age. They live in Sapporo City, Hokkaido (19 males, 13 females), Niigata City, Niigata Prefecture (59 males, 45 females), Fukuoka City, Fukuoka Prefecture (1 male, 9 females), Kasuya County, Fukuoka Prefecture (18 males, 9 females) and Kamimashiki County, Kumamoto Prefecture (9 males, 27 females). After medical screening, graded exercise tests were conducted. Heights, weights and ages are shown in Tab. 1. Furthermore, subjects understood its purpose and its merits and demerits and agreed to participate. In addition, the protocols of this investigation was approved by the ethnics committee in School of Medicine, Fukuoka University and in Graduate School of Education, Hokkaido University.

\subsection{Graded Exercise Test}

We conducted submaximal graded stepping tests using a $20 \mathrm{~cm}$ ( 8 inches) stepping platform. Exercise intensity was standardized by stepping rate (ascends/min). Stepping rate, work rate METs (Metabolic Equivalent) and number of subjects at each stage are shown in Tab. 2. In addition, work rate is shown in transfer gain to the vertical per kilogram per minute $(\mathrm{kgm} / \mathrm{kg} / \mathrm{min})$. METs was estimated from the height of the step and the number of ascends $\left(1 \mathrm{METs}=3.5 \mathrm{ml} / \mathrm{kg} / \mathrm{min}\right.$ of $\dot{\mathrm{VO}}_{2}, \dot{\mathrm{V}}_{2}$ $(\mathrm{ml} / \mathrm{kg} / \mathrm{min})=0.2 \times$ stepping rate $[$ ascends $/ \mathrm{min}]+1.33 \times$ $1.8 \times$ platform height $(\mathrm{m}) \times$ stepping rate[ascends $/ \mathrm{min}])$ [ACSM, (2000)]. Stepping rate was established at a beginning load of 10,15 or 20 ascends/min for each subject based on physical activity level, and at each 
Tab. 2. The testing protocol of the step test, and the number of participants at each stage.

\begin{tabular}{cccccc}
\hline \multirow{2}{*}{$\begin{array}{c}\text { Stepping } \\
\text { rate }\end{array}$} & $\begin{array}{c}\text { Exercise } \\
\text { intensity } \\
\text { (ascends/minute) }\end{array}$ & METs & \multicolumn{3}{c}{ Number of subjects } \\
\cline { 4 - 6 } & 3.0 & 2.9 & 80 & 38 & 42 \\
10 & 3.4 & 3.4 & 101 & 44 & 57 \\
12.5 & 3.9 & 3.9 & 152 & 80 & 72 \\
15 & 4.4 & 4.4 & 103 & 46 & 57 \\
17.5 & 4.9 & 4.9 & 202 & 103 & 99 \\
20 & 5.4 & 5.4 & 92 & 41 & 51 \\
22.5 & 5.9 & 5.8 & 170 & 87 & 83 \\
25 & 6.4 & 6.3 & 60 & 30 & 30 \\
27.5 & 6.9 & 6.8 & 67 & 42 & 25 \\
30 & 7.4 & 7.3 & 12 & 8 & 4 \\
32.5 & 7.9 & 7.8 & 4 & 3 & 1 \\
35 & & &
\end{tabular}

METs: Metabolic equivalents, estimated by means of the following equation. METs $=(0.2 \times($ stepping rate $)+1.33 \times 1.8 \times($ step height $) \times$ (stepping rate) +3.5$) / 3.5$ (ACSM 2000)

The step height was maintained at $20 \mathrm{~cm}$.

stage was increased by 2.5 ascends/min. However, in the Niigata City test, the workload increase rate was 5 times/min per stage. Exercise duration was 4 $\mathrm{min} / \mathrm{stage}$, and a $2 \mathrm{~min}$ rest period was established between stages. We measured LA and HR at rest, HR for $30 \mathrm{sec}$ before conclusion of each work stage, LA and rating of perceived exertion (RPE) immediately after conclusion of each work stage. LA was measured by taking $5: \mu \ell$ of blood from the earlobe and measuring with a portable blood lactate measuring device, Lactate Pro (Arkray, Japan) [Pyne, et al., (2000)]. Further, This research assumed LA below $0.8 \mathrm{mmol} / 1$, which the Lactate Pro cannot measure, as $0.7 \mathrm{mmol} / \mathrm{l}$. Also, HR and RPE were measured with an HR monitor, Accurex Plus (Polar Electric, Finland) and the Borg Scale. The step test was finished according the desires of subjects, and was conducted within the exercise intensity of which subjects can maintain the prescribed stepping rate safely, although $4 \mathrm{mmol} / \mathrm{l}$ of LA and 15 of RPE were established as the standard of termination criteria.

\subsection{Assessment of parameters associated with blood lactate concentration}

In the graded exercise test, for 23 males and 29 females (total 52) who met the following criteria, we determined exercise intensity corresponding to LT and fixed LA (exercise intensity equivalent to blood lactate concentration at rest [LAr]. $\mathrm{LAr}+0.1 \mathrm{mmol} / 1, \mathrm{LAr}+0.2 \mathrm{mmol} / 1, \mathrm{LAr}+0.3$ $\mathrm{mmol} / 1, \mathrm{LAr}+0.4 \mathrm{mmol} / 1, \mathrm{LAr}+0.5 \mathrm{mmol} / \mathrm{l}, \mathrm{LAr}+1$ $\mathrm{mmol} / \mathrm{l}$ and $2 \mathrm{mmol} / \mathrm{l}$ : hereafter, respectively, METs@LAr,METs@LAr+0.1, METs@LAr+0.2,
METs@LAr+0.3, METs@LAr+0.4, METs@LAr+0.5, METs@LAr+1 and METs@LA2). (1) Two or more LA-samples were less than LAr+0.5 mmol/l. (2) Two or more LA-samples were higher than $2 \mathrm{mmol} / \mathrm{l}$. (3) The difference in LT among 3 observers was less than $10 \%$. In addition, these 52 subjects were drawn from 105 subjects (47 males, 58 females) with a gradual load increase of 2.5 ascends/min. Of these 105 people, the number that could not meet Criterion (1) and (2) was 13 subjects (12\%, 4 males, 9 females) and 31 subjects $(30 \%, 14$ males, 17 female), respectively. Of the 66 subjects who met Criteria (1) and (2) (30 males, 36 females), these variables were determined for the 52 subjects ( 23 males, 29 females) who met Criterion (3).

\subsubsection{Lactate Threshold (Fig.1.-a)}

We determined LT by the method of Beaver et al. (1985) (Fig.1.-a). That is, we calculated the regression by the minimum squares method, dividing exercise load into pre-LT and post-LT by observation from a graph drawn as log-exercise intensity for the independent variable and log-LA for the dependent variable.

(1) $\log \mathrm{LA}=$

$a_{1} \times \log$ exercise intensity $+b_{1}$ (regression pre-LT)

(2) $\log \mathrm{LA}=$

$a_{2} \times \log$ exercise intensity $+b_{2}$ (regression post-LT)

LT was considered to be at the intersection of the two lines, and was calculated by the following formula.

LT $(\log$ exercise intensity $)=\left(b_{1}-b_{2}\right) /\left(a_{2}-a_{1}\right)$

\subsubsection{Determination of exercise intensity corresponding to fixed LA by standard assessment} (Fig. 1.-b)

We estimated METs@LAr,METs@LAr+0.1, METs@LAr+0.2, METs@LAr+0.3, METs@LAr+0.4, METs@LAr+0.5,METs@LAr+1 and METs@LA2 by formula (2) (standard assessment, Fig. 1.-b). Further, these LA was confirmed to be the standard for LT assessment. [Beaver et al., (1985, 1986); Coyle et al., (1983); Hughson and Green, (1982); Kinderman et al., (1979)].

\subsubsection{Determination of exercise intensity corresponding to fixed LA by simple assessment} (Fig. 1.-c)

As a simple assessment of exercise intensity corresponding to fixed LA, we determined METs@LAr, 

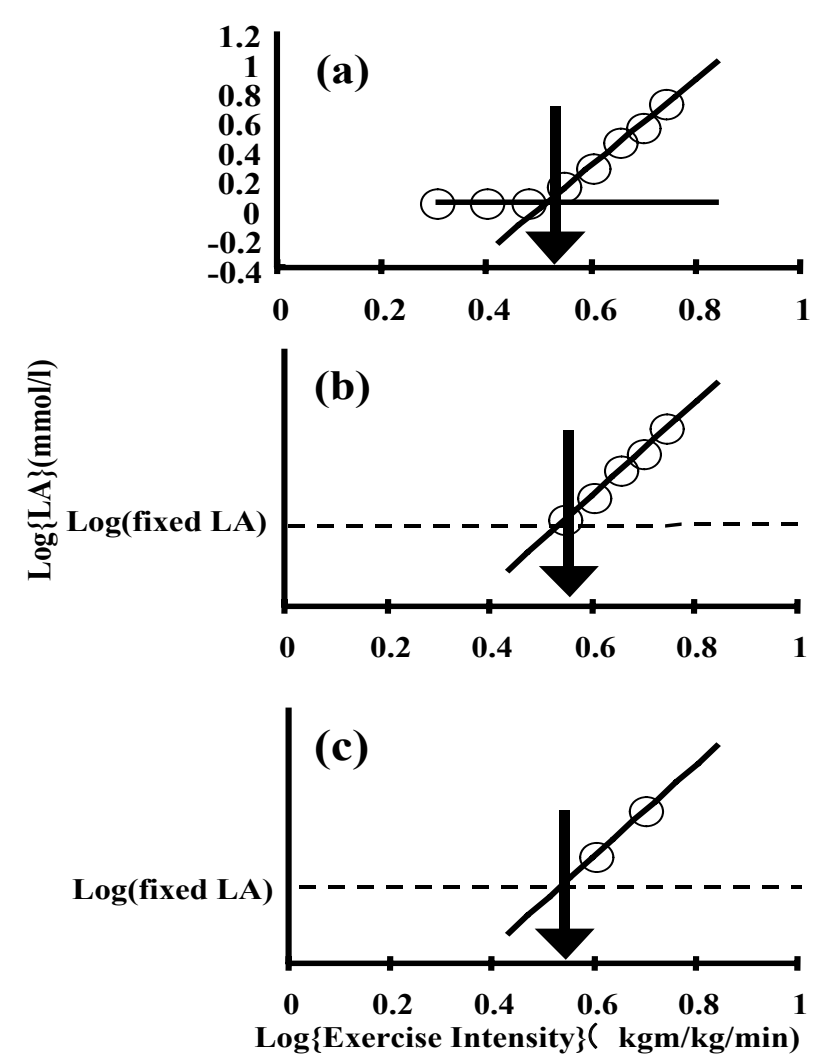

Fig. 1. Graphing procedure for determining the parameters associated with the blood lactate concentration.

(a)The upper illustrate shows the procedure for determining lactate threshold (LT). (b)The centers illustrate shows the standard assessment of exercise intensity at fixed LA levels. (c) The lower illustrate shows the simple assessment of the exercise intensity at fixed LA value. Arrows $(\downarrow)$ show LT and the exercise intensity of which correspond to the each target LA, respectively. The dotted lines $(\ldots)$ show the target LA levels. The solid lines $(-)$ show the regression line between exercise intensity and LA.

METs@LAr+0.1, METs@LAr+0.2, METs@LAr+0.3, METs@LAr+0.4, METs@LAr+0.5,METs@LAr+1 and METs@LA2 by means of 2 LA and exercise intensities. For this assessment, two pairs of exercise intensify and LA of which corresponded to bellow and above $2 \mathrm{mmol} / \mathrm{l}$ of LA were drawn from 2, 3, 4, 5, 6 and $7 \mathrm{kgm} / \mathrm{kg} / \mathrm{min}$ during the graded exercise test. Exercise intensities corresponding to each LA were converted to their logarithms and estimated according to the linear formula obtained from these samples. (simple assessment; Fig. 1.-c).

\subsection{Statistics}

F-test and paired T-test were used to determine the difference among LT and alternative methods. Furthermore, we studied agreement among methods using Bland and Altman plots (1986), employing the $95 \%$ interval of deviation (average value \pm 2 standard deviation). Analysis of correlation between methods was conducted using the Pearson (r). All
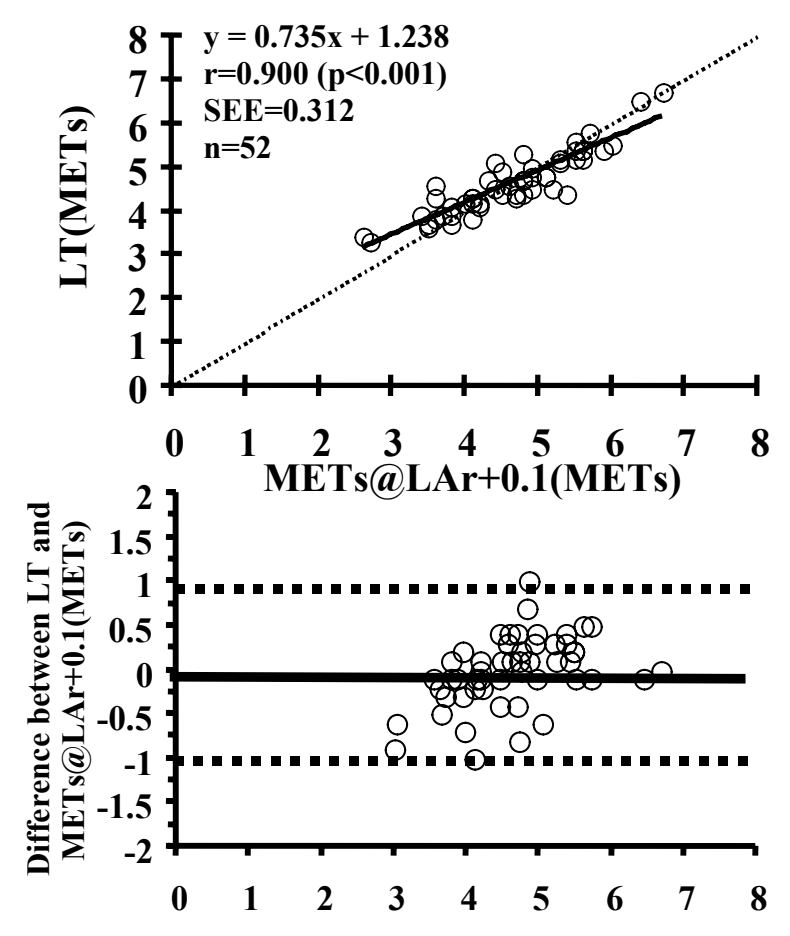

Mean of LT and METs@LAr+0.1(METs)

Fig. 2. The comparison of the lactate threshold and the simple assessment of METs@LAr+0.1. LT: lactate threshold, METs@LAr+0.1: METs at which LA increased $0.1 \mathrm{mmol} / 1$ from resting level, determined by the simple assessment. In the upper graph, the dotted line $(\ldots)$ is the identity line $(\mathrm{y}=\mathrm{x})$. The lower graph shows the Bland and Altman plots. The plots show the difference between LT and METs@LAr+0.1 (METs@LAr+0.1 minus LT). The solid line $(-)$ shows the mean of differences. The dotted lines (...) show the 2 standard deviations of differences.

measurement values in this paper are shown in average values and standard deviation (average value \pm standard deviation). $\mathrm{p}<0.05$ was taken to indicate a statistical significance.

\section{Results}

LA, ऍّA (increase from LA at rest) and METs atLT,METs@LAr+0.1,METs@LAr+0.2, METs@LAr+0.3, METs@LAr+0.4, METs@LAr+0.5,METs@LAr+1 and METs@LA2 estimated by standard assessment are shown on Tab. 3. At LT, LA was $1.2 \pm 0.3 \mathrm{mmol} / \mathrm{l}$, and $\triangle \mathrm{LA}$ was $0.1 \pm 0.3 \mathrm{mmol} / 1$. METs $@ \operatorname{LAr}(4.5 \pm 0.8 \mathrm{METs})$ was a significantly lower compared to LT $(4.6 \pm 0.7$ METs $)(p<0.05)$. In addition, METs@LAr+0.2, METs@LAr+0.3,METs@LAr+0.4, METs@LAr+0.5, METs@LAr+1 and METs@LA2 $(4.8 \pm 0.8,4.9 \pm 0.8,5.0 \pm 0.8,5.1 \pm 0.8,5.6 \pm 0.8$ and $5.5 \pm$ 0.8 METs) were significantly higher compared to LT $(p<0.01)$. On the other hand, we could not confirm a 
Tab. 3. The blood lactate concentration and MET value at LT and fixed blood lactate levels.

\begin{tabular}{lccccccccc}
\hline & LT & LAr & LAr+0.1 & LAr+0.2 & LAr+0.3 & LAr+0.4 & LAr+0.5 & LAr+1 & LA2 \\
\hline LA(mmol/l) & $1.2(0.3)$ & $1.1(0.2) \#$ & $1.2(0.2)$ & $1.3(0.2) \# \#$ & $1.4(0.2) \# \#$ & $1.5(0.2) \# \#$ & $1.6(0.2) \# \#$ & $2.1(0.2) \# \#$ & 2.0 \\
$\Delta$ LA(mmol/1) & $0.1(0.3)$ & 0 & 0.1 & 0.2 & 0.3 & 0.4 & 0.5 & 1.0 & $0.9(0.2) \# \#$ \\
METs & $4.6(0.7)$ & $4.5(0.8) \#$ & $4.7(0.8)$ & $4.8(0.8) \# \#$ & $4.9(0.8) \# \#$ & $5.0(0.8) \# \#$ & $5.1(0.8) \# \#$ & $5.6(0.8) \# \#$ & $5.5(0.8) \# \#$ \\
Deviation(METs) & - & $-0.1(0.3)$ & $0.0(0.3)$ & $0.1(0.3)$ & $0.3(0.3)$ & $0.4(0.3)$ & $0.5(0.3)$ & $1.0(0.4)$ & $0.9(0.4)$ \\
Deviation(\%) & - & $-2.4(7.0)$ & $0.4(6.8)$ & $3.0(6.7)$ & $5.6(6.6)$ & $8.1(6.7)$ & $10.5(6.8)$ & $21.1(8.3)$ & $19.2(9.2)$ \\
Pearson(r) & - & $0.930^{*}$ & $0.930^{*}$ & $0.929^{*}$ & $0.927^{*}$ & $0.924^{*}$ & $0.919^{*}$ & $0.888^{*}$ & $0.865^{*}$ \\
\hline
\end{tabular}

$\mathrm{n}=52$

Data was expressed as mean (standard deviation).

LT:lactate threshold.

LAr : resting level

$\mathrm{LAr}+0.1$ to $\mathrm{LAr}+1: 0.1$ to $1 \mathrm{mmol} / 1$ increased from resting level, respectively.

LA2 : $2 \mathrm{mmol} / \mathrm{l}$.

The METscorresponding to each fixed value were determined by means of LA and exercise intensity ranged above the LT.

METs: metabolic equivalents.

$\triangle \mathrm{LA}(\mathrm{mmol} / \mathrm{l})$ shows the increments of LA from resting level.

Deviation shows the difference in the MET value between the LT and the fixed LA levels (METs at fixed LA levels minus METs at LT).

Pearson(r) shows the correlation between the MET value at LT and the that at fixed LA levels.

\#,\#\# There was a significant difference, compared with that at LT(\# $\mathrm{p}<0.05$, \#\# $\mathrm{p}<0.01)$.

* The correlation was significant $(\mathrm{p}<0.01)$.

significant difference between METs@LAr+0.1 (4.7 \pm 0.8 METs) and LT. Moreover, correlation between METs@LAr+0.1 and LT $(\mathrm{r}=0.930, \mathrm{p}<0.001)$ and the limit of agreement $(0.0 \pm 0.6 \mathrm{METs})$ were better than other alternative assessments.

METs@LAr, METs@LAr+0.1, METs@LAr+0.2, METs@LAr+0.3, METs@LAr+0.4, METs@LAr+0.5, METs@LAr+1 and METs@LA2 estimated by simple assessment were $4.5 \pm 0.8,4.7 \pm 0.8,4.8 \pm 0.8,4.9 \pm$ $0.8,5.0 \pm 0.8,5.1 \pm 0.8,5.6 \pm 0.8$ and $5.5 \pm 0.8$ METs, respectively, and there was no significant difference in METs value between 2 assessments. Also, as well as the standard assessment, METs@LAr+0.1 was not significantly different compared with LT. Furthermore a significant difference could not be confirmed between LT and METs@LAr+0.2, METs@LAr+0.3, METs@LAr+0.4, METs@LAr+0.5, METs@LAr+1 and METs@LA2 estimated by simple assessment. Between METs@LAr+0.1 assessed by the simple assessment and LT, the correlation (Fig. 2.; $\mathrm{r}=0.900$, $\mathrm{p}<0.001)$ deviation $(0.0 \pm 0.3 \mathrm{METs})$ and the limit of agreement $(0.0 \pm 0.6 \mathrm{METs})$ were all not inferior to the those between LT and standard assessment.

Fig. 3. shows distribution of exercise intensity at which LA exceeded $2 \mathrm{mmol} / \mathrm{l}$. In both men and women, the highest frequency of exercise intensity at which $2 \mathrm{mmol} / \mathrm{l}$ or greater of LA appeared was $5 \mathrm{kgm} / \mathrm{kg} / \mathrm{min}, 45 \%$ of males $(\mathrm{n}=48)$ and $53 \%$ of females $(n=55)$. Additionally, in $90 \%$ of male $(n=97)$ and $92 \%$ of female $(\mathrm{n}=95)$, LA exceeded $2 \mathrm{mmol} / \mathrm{l}$ at intensities of 3.5 to $6 \mathrm{kgm} / \mathrm{kg} / \mathrm{min}$. Thus, in these 90\% of subjects, METs@LAr +0.1 is successfully determined during exercise intensity of 3.5 to 6 $\mathrm{kgm} / \mathrm{kg} / \mathrm{min}$, because LA lower and higher than $2 \mathrm{mmol} / \mathrm{l}$ were obtained. Among the remaining subjects, those whose LA exceeded $2 \mathrm{mmol} / \mathrm{l}$ at 2.5 or $3 \mathrm{kgm} / \mathrm{kg} / \mathrm{min}$ constituted $7 \%$ of males $(\mathrm{n}=7)$ and $4 \%$ of women $(n=4)$. Those subjects whose LA was less than $2 \mathrm{mmol} / \mathrm{l}$ at $6 \mathrm{kgm} / \mathrm{kg} / \mathrm{min}$ constituted $3 \%$ of males $(n=3)$ and $4 \%$ of women $(n=4)$.

\section{Discussion}

The results of this investigation clearly indicate the validity of METs@LAr+0.1 estimated from bench stepping test at step rates of 15, 20, 25 and 30 ascends/minute using a $20 \mathrm{~cm}$ platform as a simple assessment of LT in older population.

Although LT is evaluated as the exercise intensity at which LA begins to increase acceleratory from resting level, some researches have been conducted using exercise intensity equivalent to LA increased to $1 \mathrm{mmol} / \mathrm{l}$ from resting level, or a fixed LA of 2 mmol/l, etc. [Coyle et al., (1983) ; Dickstein et al., (1990) ; Hughson and Green, (1982); Kinderman et al. (1979)]. However, exercise intensity equivalent to these LA has been indicated higher compared to LT [Yoshida et al., (1987) ; Dickstein et al., (1990)]. In this research, LA at LT was $1.2 \pm 0.3 \mathrm{mmol} / \mathrm{l}$, and the increase from resting level was $0.1 \pm 0.3 \mathrm{mmol} / \mathrm{l}$. As a result, LT was higher compared to exercise intensity equivalent to resting level of LA, and a low value compared to exercise intensity equivalent to LA that has increased $0.2 \mathrm{mmol} / 1$ or more from resting 

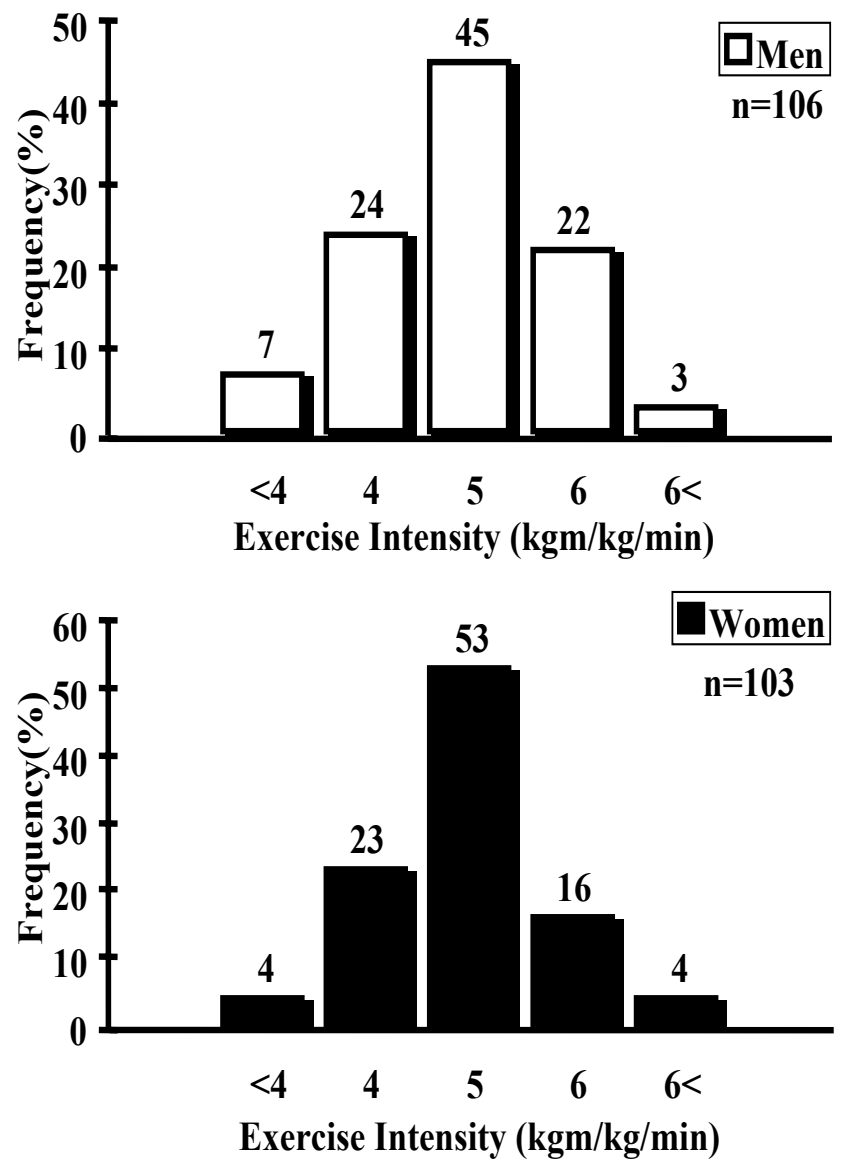

Fig. 3. The frequency distributions of exercise intensity at which LA exceeded $2 \mathrm{mmol} / \mathrm{l}$.

level. In contrast, $0.1 \mathrm{mmol} / 1$ above resting level of LA did not confirm the significantly difference in corresponding exercise intensity compared with LT. We have already founded that the increase of LA from resting level at LT approximated to $0.1 \mathrm{mmol} / 1$ in younger individuals (unpublished data). Also, Beaver et al. (1985) have also reported that LA at LT approximated to resting level in younger people. Therefore, LA at LT is apparently lower than LA as used to date, and $0.1 \mathrm{mmol} / \mathrm{l}$ above resting level would be appropriate.

In older individuals, previously described procedures for determining LT that evaluate accelerate increase of LA based on the frequently blood sampling [Beaver et al., (1985) ; Tokmakidis et al., (1998)] was sometime cause the lower accuracy and/or the determination rate, because of the difficulties in blood sampling above LT [Belman and Gaesser, (1992)]. In this research, similarly, of the 105 subjects who were given the graded exercise test, we were able to obtain sufficient LA to determine LT in only about half, or 52 subjects. In contrast, use of the simple assessment proposed in this research will permit a dramatic improvement in LT determination rate, because the criteria of present procedure is $2 \mathrm{mmol} / \mathrm{l}$ of LA. In addition, since the number of samplings, including the baseline measurement, will only be 3 to 5 times, the subject's burden and the various effort associated with the measurements will be greatly reduced. If the Lactate-Pro [Pyne et al., (2000)] is used, the cost per measurement will be about $¥ 1,000$ (US\$9.00) (not including cost of the Lactate-Pro. Total for disposables, such as stripes and knives etc.).

Results of this research revealed that a stepping test from 3 to $6 \mathrm{kgm} / \mathrm{kg} / \mathrm{min}$ is an appropriate exercise protocol for determining LT in older people. In order to determine METs@LAr+0.1, LA must be measured at bellow and above $2 \mathrm{mmol} / \mathrm{l}$. As shown in Fig. 3., in $90 \%(n=97)$ of men and $92 \%(n=95)$ of women, LA reached $2 \mathrm{mmol} / 1$ during step test of 3.5 to 6 $\mathrm{kgm} / \mathrm{kg} / \mathrm{min}$. Thus the step test ranged from 3 to 6 $\mathrm{kgm} / \mathrm{kg} / \mathrm{min}$ with the termination criteria as $2 \mathrm{mmol} / \mathrm{l}$ of LA will successfully determine LT in almost $90 \%$ of the community dwelling elderly. However, this protocol failed to determine LT in about $10 \%$ of the subjects. In regard to the elderly people for whom LT could not be determined, in $7 \%$ of men $(n=7)$ and $4 \%$ of women $(\mathrm{n}=4)$, LA exceeded $2 \mathrm{mmol} / \mathrm{l}$ at 3 $\mathrm{kgm} / \mathrm{kg} / \mathrm{min}$ or more light intensity. On the other hand, in $3 \%$ of men $(n=3)$ and $4 \%$ of women $(n=4)$, LA did not exceed $2 \mathrm{mmol} / \mathrm{l}$ at $6 \mathrm{kgm} / \mathrm{kg} / \mathrm{min}$. In the former, LT was estimated to be bellow 3 METs and/or the general walking, thus they should exercise under the supervised condition as well as patients. The latter, on the other hand, have aerobic capacity equal to or greater compared with younger individuals. Thus, we concluded that stepping tests of $15,20,25$ and 30 ascends/min using a $20 \mathrm{~cm}$ platform constitute appropriate exercise intensity for determining LT in the community dwelling elderly with normal aerobic capacity.

In this research, we proposed a method using stepping tests in order to simplify a graded exercise tests. While the stepping test cannot be applied to individuals who have balance problem or are extremely deconditioned, little or no equipment, the easily for transportable of steps, usual exercise mode are the merits of step test compared with other ergometers. [ACSM, (2000)]. The previously discussed problem about the disdesireble accuracy in evaluation of aerobic capacity based on HR response [Cox et al. (1992); Sidney and Shephard (1977); 
Takeshima et al., (1992)] would be improved by employing LA measurement [Bell and Jacobs (1992a 1992b)]. Furthermore it has been suggested that older individuals could perform the step test using a $20 \mathrm{~cm}$-platform safely without major abnormal ECG. [Furuna, et al., (1994)].

Moreover, using LT determined in the stepping tests directly as the training intensity is a major merit. Additionally, older people may have to avoid the exercise under such inclement conditions as rain or summer heat in southern climates and winter cold in northern climates, therfore the indoor bench stepping exercise that elderly can perform in preferable condition should be reccomended for older adults. In addition, stepping exercise is a form of squatting exercise, so it not only provides aerobic exercise, it also has great possibilities as an effective training exercise for the large anterior femoral muscles and the large pelvic muscles, which are prone to atrophy in older people. In other words, the pesent procedure will promote the primary care for older individuals, because the bench stepping training is paractical and effective on both the cardiovascular disease and the leg failure especially in thigh.

Results of this research showed that 15, 20, 25 and 30 ascends/min of stepping rates seem to be appropriate intensity of step test using a $20 \mathrm{~cm}$-platform for a simple assessment of LT in community dwelling older individuals. However, the validity of these exercise intensity was obtained in limited subjects, therefore this method may have to be re-confirmed in other groups. Thus, the next step is to examine the appropriate exercise intensity corresponding to bellow and above $2 \mathrm{mmol} / \mathrm{l}$ of LA in variety conditioned individuals, in order to improve the usefulness of this assessment.

\section{Conclusion}

We conducted the basic investigation with the purpose of developing a simple assessment of aerobic capacity and exercise prescription which can be used in the health promotion fields for older individuals. Consequently, the determination of exercise intensity of which LA increased $0.1 \mathrm{mmol} / \mathrm{l}$ above baseline level by the step test at 15,20,25 and 30 ascends/minute of stepping rate, using a $20 \mathrm{~cm}$ platform was valid as the simple assessment of LT in older population.

\section{Acknowledgment}

This investigation was supported by the Special Coordination Funds of the Ministry of Education, Culture, Sports, Science and Technology, the Japanese government. Furthermore authors have special thanks for the member of the Laboratory of Exercise Physiology in Fukuoka University and Laboratory of Human Performance and Fitness, Graduate School of Education in Hokkaido University.

\section{References}

American College of Sports Medicine, (2000) ACSM's guidelines for exercise testing and prescription, 6th Ed., The Williams \& Wilkins Company, Baltimore.

Beaver, W.L., Wasserman, K., Whipp B.J. (1985) Improved detection of lactate threshold during exercise using a log-log transformation. J Appl Physiol. 59: 1936-1940.

Beaver, W.L., Wasserman K., Whipp B.J. (1986) A new method for detecting anaerobic threshold by gas exchange. J Appl Physiol. 60: 2020-2027.

Bell, D.G., \& Jacobs,I. (1992a) Blood lactate response to the Canadian aerobic fitness test (CAFT). Can J Spt Sci. 17: 14-18.

Bell, D.G., \& Jacobs, I. (1992b) Blood lactate response to the Canadian aerobic fitness test in females. Can J Spt Sci. 17: 148-151.

Belman, M.J., \& Gaesser, G. A. (1991) Exercise training bellow and above the lactate threshold in the elderly. Med Sci Sports Exerc. 23: 562-568.

Bland, J. M., \& Altman, D.G. (1986) Statistical methods for assessing agreement between two methods of clinical measurement. Lancet. 8476: 307-310.

Coyle, E.F., Martin, W.H., Ehsani, A., Hgberg, J.M., Bloomfield, S.A., Sinacore, D.R., Holloszy, J.O. (1983) Blood lactate threshold in some well trained heart disease patients. J Appl Physiol. 54: 18-23.

Cox, M.H., Thomas, S.G., Weller, I.M.R., Corey, P. (1992) Reliability and validity of a fitness assessment for epidemiological studies. Can J Spt Sci. 17: 49-55.

Dickstein, K., Barvik, S., Aarsland., T., Snapinn, S., Karlsson, J., (1990) A comparison of methodologies in detection of anaerobic threshold. Circulation. 80: 38-46.

Furuna, T., Itoh, H., Nagasaki, H., Fujisawa, A., Niimi, N., Maruyama, H., Kinigasa, T. (1994) Effects of aging on the aerobic capacity measured by a step-test. Jpn J Geriat. 31:45-51. (in Japanese)

Katamoto, S., (1980) Indirect measurement of maximal oxygen uptake. Journal of Health, Physical Education and Recreation. 30: 823-827. (in Japanese)

Hughson, R.L., \& Green, H.J. (1982) Blood acid-base and lactate relationships studied by ramp work tests. Med Sci Sports Exerc. 14:297-302.

Kinderman, W., Simon, G., Keul, J. (1979) The significance of the aerobic-anaerobic transition for the determination of work load intensities during endurance training. Eur J Appl Physiol. 42: 25-34.

Kiyonaga, A., Arakawa, K., Tanaka, H., Shindo, M. (1985) Blood pressure and hormonal responses to aerobic exercise. Hypertension. 7:125-131.

Motoyama, M., Sunami, Y., Kinoshita, A., Kiyonaga, A., Arakawa, K., Tanaka, H., Shindo, M., Irie, T., Urata, H., Sasaki, J., Arakawa, K., (1998) Blood pressure lowering effect of low intensity aerobic training in elderly hypertensive patients. Med Sci Sports Exerc. 30:818-823. 
Paterson, D.H., Cunninghan, D.A., Koval, J.J., Croix, C.M.T.(1999) Aerobic fitness in a population of independently living men and women aged 55-86 years. Med Sci Sports Exerc. 31:1813-1820.

Pyne, D.B., Boston, T., Martin, D.T., Logan, A. (2000) Evaluation of the lactate pro analyzer. Eur. J. Appl. Physiol. 82:112-116.

Sidney, K.H., \& Shephard, R.J. (1977) Maximum and sub-maximum exercise tests in men and women in seventh, eight, and ninth decades of life. J. Appl. Physiol. 43: 280-287.

Sunami, Y.,Motoyama, M.,Kinoshita, F.,Mizooka, Y.,Sueta, K.,Matsunaga, A.,Sasaki, J.,Tanaka, H.,Shindo, M. (1999) Effects of low intensity aerobic training on the high-density lipoprotein cholesterol concentration in healthy elderly subjects. Metabolism. 48: 984-988

Takeshima, N., Tanaka, K., Kobayashi, A., Watanabe, A., Sumi, K., Koto T. (1992) Validity of the maximal aerobic capacity estimated from submaximal cycling exercise and field performance test in the elderly. Jpn J Phys Fitness Sports Med. 41: 295-303.(in Japanese)

Tokmakidis, S.P., Leger L.A., Pilianidis, T.C. (1998) Failure to obtain a unique threshold on the blood lactate concentration curve during exercise. Eur J Appl Physiol. 77: 333-342.

Yoshida, T., Chida, M., Ichioka, M., Suda, Y. (1987) Blood lactate parameters related to aerobic capacity and endurance performance. Eur J Appl Physiol. 56: 7-11.

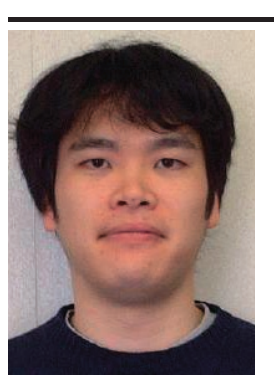

Name:

Makoto Ayabe

\section{Affiliation:}

Doctoral Program, Graduate School of Education, Hokkaido University

\section{Address:}

Nishi 7, Kita 11-jo, Kita-Ku, Sapporo City 060-0811 Japan

Brief Biographical History:

1999 - Master's Program, Graduate School of Physical Education, Fukuoka University

2001 - Doctoral Program, Graduate School of Education,

Hokkaido University

Membership in Learned Societies:

- Japanese Society of Physical Education, Health and Sports Science

- The Japanese Society of Physical Fitness and Sports Medicine - Japan Society of Exercise and Sport Physiology 


\section{APPENDIX}

\section{The procedure for the simple assessment of lactate threshold}

\section{A. The testing protocol}

\begin{tabular}{lccccccc}
\hline $\begin{array}{l}\text { Stage } \\
\text { (minutes) }\end{array}$ & $\begin{array}{c}\text { Time } \\
\text { (ascends/min) }\end{array}$ & \multicolumn{3}{c}{\begin{tabular}{c} 
Stepping Rate \\
\cline { 5 - 7 }
\end{tabular}} & METs & \multicolumn{3}{c}{ Measurement } \\
\hline Rest & - & - & - & $\bigcirc$ & $\bigcirc$ & $\times$ \\
1 & $0-4$ & 15 & 3.9 & $\bigcirc$ & $\bigcirc$ & $\bigcirc$ \\
2 & $6-10$ & 20 & 4.9 & $\bigcirc$ & $\bigcirc$ & $\bigcirc$ \\
3 & $12-16$ & 25 & 5.9 & $\bigcirc$ & $\bigcirc$ & $\bigcirc$ \\
4 & $18-22$ & 30 & 6.9 & $\bigcirc$ & $\bigcirc$ & $\bigcirc$ \\
\hline
\end{tabular}

METs: Metabolic equivalents, estimated by means of the following equation. METs $=(0.2 \times($ stepping rate $)+1.33 \times 1.8 \times($ step height $) \times($ stepping rate) +3.5$) \times 3.5^{-1}$ (ACSM 2000). The step height is maintained at $20 \mathrm{~cm}$ (8 inches). LA: blood lactate concentration, should be measured at rest and following each stage. HR: heart rate, should be measured at rest and the final 30 seconds of each stage. RPE: rating of perceived exertion, should be measured following each stage.

\section{Test Termination}

- All subjects should be encouraged to continue the test until LA exceeds $2 \mathrm{mmol} / \mathrm{l}$.

\section{Caution}

-In subjects, those who LA exceeded $2 \mathrm{mmol} / \mathrm{l}$ at the first stage, LT is unable to be determined. These low-fit individuals should finish the test immediately, and perform the standard graded exercise test.

-In subjects, those who LA did not exceeded $2 \mathrm{mmol} / \mathrm{l}$ by the end of this test, LT is unable to be determined. These high-fit individuals should perform this test again by means of a higher step.

\section{B. $L$ T determination}

\section{$\log -\mathbf{L A}(\mathbf{m m o l} / \mathbf{l})$}

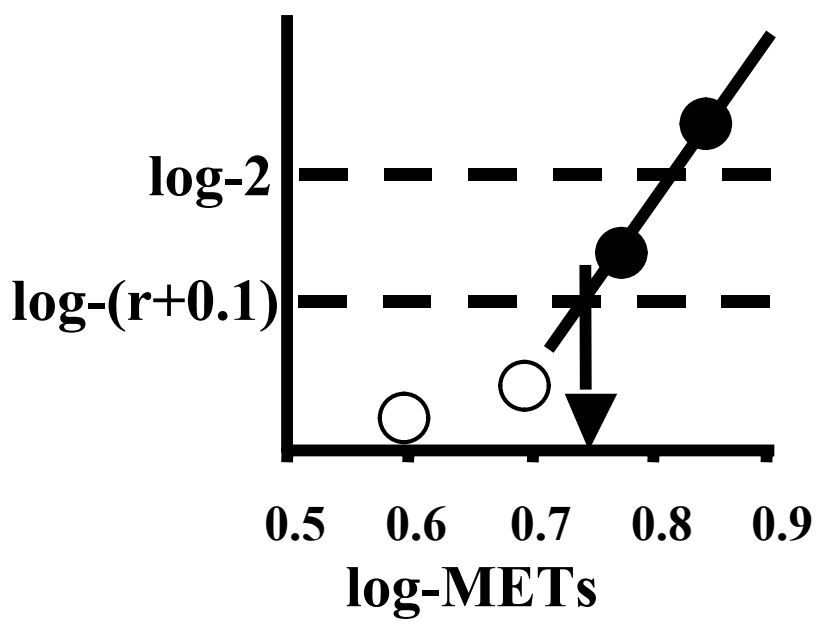

The dotted lines (---) show the LA corresponding to $\log -2 \mathrm{mmol} / \mathrm{l}$ (upper) and log- $0.1 \mathrm{mmol} / 1$ above baseline level (lower), respectively. The closed circles $(-)$ show the LA ranged bellow and above 2 $\mathrm{mmol} / \mathrm{l}$. The solid line (-) shows the regression line obtained from log-transformed these samples. The other samples (open circles: $\bigcirc$ ) do not use for this analysis. An arrow $(\downarrow)$ shows the LT. In this participants, man aged 65 years old, LT was 5.6 METs (log-0.75 METs). 